\title{
A Two-Component Model of the HE Radiation from LS 5039 as observed by Fermi/LAT
}

\author{
Maria Kapala * \\ University of Warsaw \\ E-mail: mkapala@astrouw.edu.pl \\ Tomasz Bulik \\ University of Warsaw, CAMK \\ E-mail: tbeastrouw.edu.pl

\section{Bronislaw Rudak} \\ CAMK \\ E-mail: bronek@ncac.torun.pl
}

\section{Guillaume Dubus}

$L A O G$

E-mail: guillaume.dubuscobs.ujf-grenoble.fr

\section{Michal Lyczek}

ICM

E-mail: m. lyczekeicm.edu.pl

We aim at explaining the Fermi LAT data for LS 5039 by including a rotation powered pulsar. The pulsar plays a two-fold role in generating the HE emission in the system. We show that with a few modest assumptions about the pulsar one can reproduce both the spectrum and the orbital modulations.

25th Texas Symposium on Relativistic Astrophysics

December 6-10, 2010

Heidelberg, Germany

\footnotetext{
* Many thanks to my collaborators
} 


\section{Assumptions}

Key ingredients of the model:

- middle-aged pulsar (magnetospheric radiation)

- companion O-star (source of soft photons)

- collimated pulsar wind of unshocked electrons

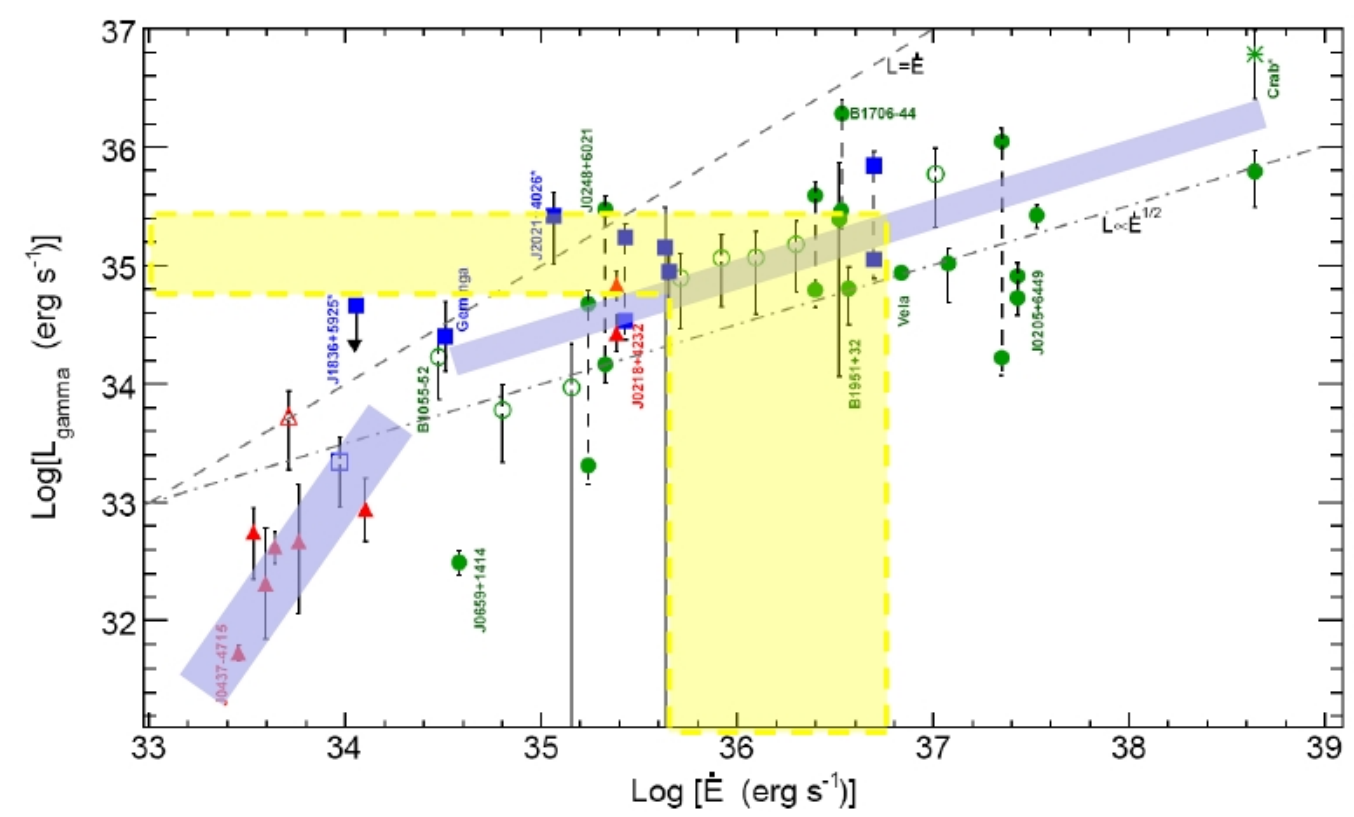

Figure 1: Semi-empirical relations between the spin-down power E and the luminosity LPSR in $\gamma$ - rays. Based on Fermi LAT pulsars taken from Abdo et al. (2010b) [2].

The model assumes that the Fermi LAT flux is due to two components: a pulsar and an Inverse Compton Scattering (ICS) component due to the wind. The inferred $\gamma$-ray luminosity of the pulsar component a level of $\sim 10^{35} \mathrm{erg} / \mathrm{s}$. Yellow rectangles in Fig. 1 show how this pseudo-luminosity is used to estimate the spin-down luminosity which falls in the range of $10^{36} \mathrm{erg} / \mathrm{s}$ to $10^{37} \mathrm{erg} / \mathrm{s}$. The upper limit comes from the age of its O-star companion $\left(10^{5} y r\right)$ and the lower from the age of young Crab-like pulsars $\left(10^{3} y r\right)$.

\section{Binary system}

The distance to LS 5039 is $\sim 2.5 \mathrm{kpc}$. The orbit of the system is elliptical with the eccentricity 0.35 , the semi-major axis $\sim 0.2 \mathrm{AU}$ and the inclination $60^{\circ}$, the latter is assumed. We also assume that the companion O-star is a point-like source of monoenergetic photons of $3 \mathrm{eV}$. The star is also a source of a strong wind which interacts with the pulsar magnetospheric wind in a similar way as proposed by Dubus et al (2010) [7]. 


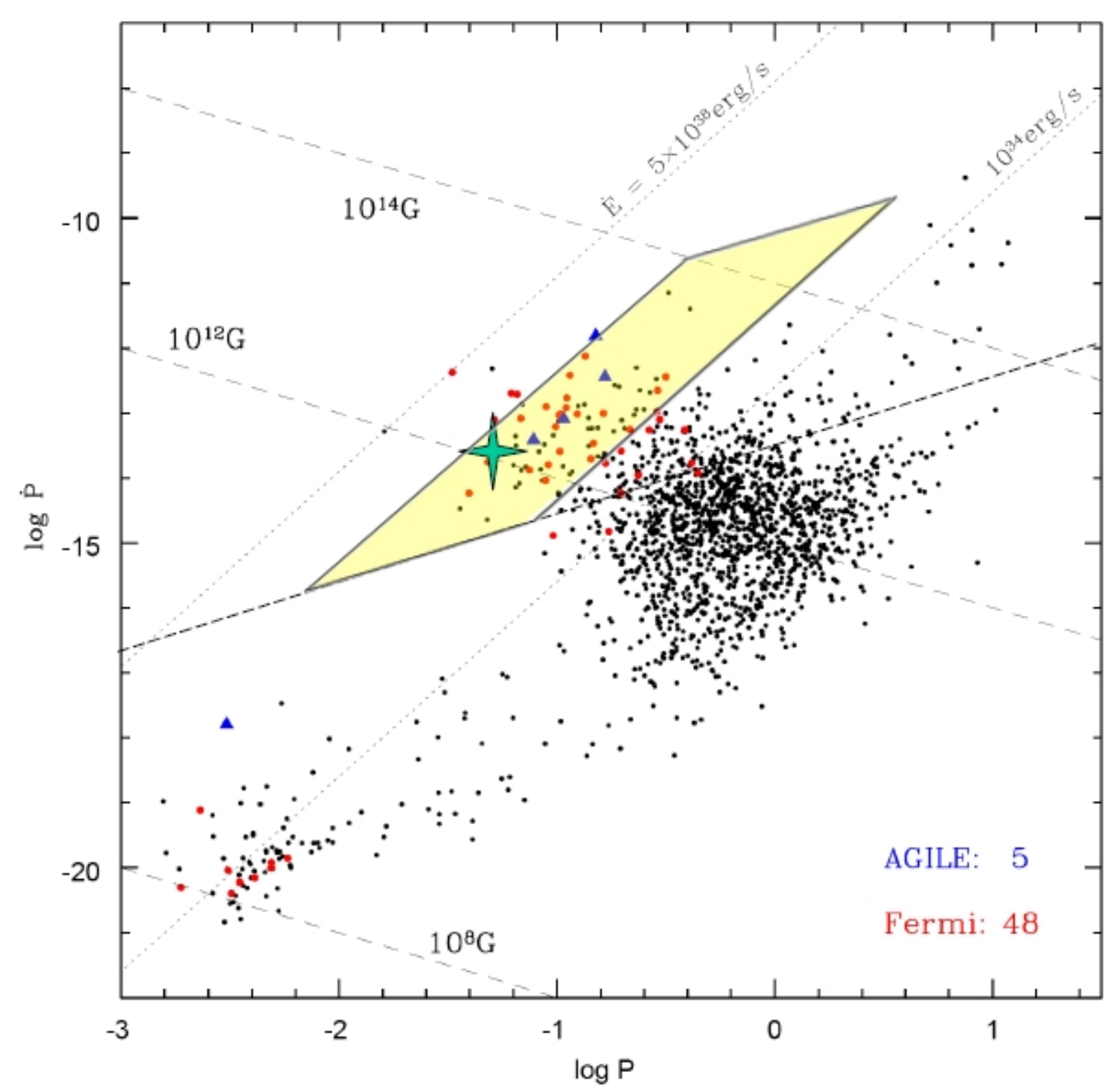

Figure 2: The yellow rhombus shows the region where the putative LS 5039 pulsar may reside. The position of a PSR B1259-63 is indicated with green star for comparison.

\section{Results}

The pulsar contributes to the HE radiation from the system in two different ways, resulting in two spectral components. The first component is due to (pulsed) emission originating in magnetospheric outer gaps of the pulsar. It picks around $1 \mathrm{GeV}$. Its amplitude does not change with the orbital phase. The second component is due to Inverse Compton Scattering of the O-star photons by electrons supplied to the radial unshocked outflow by the pulsar. The electrons are assumed to tap a major fraction of their energy from the Poynting flow. For most of the orbital cicle this component dominates at $100 \mathrm{MeV}$ over the pulsar's contribution. Fig. 4 shows both components and their sum after averaging over the orbital period. The model spectrum is harder in the range $100 \mathrm{MeV}-1 \mathrm{GeV}$ than the Fermi/LAT spectrum. This is due to the simplified point-like treatment 


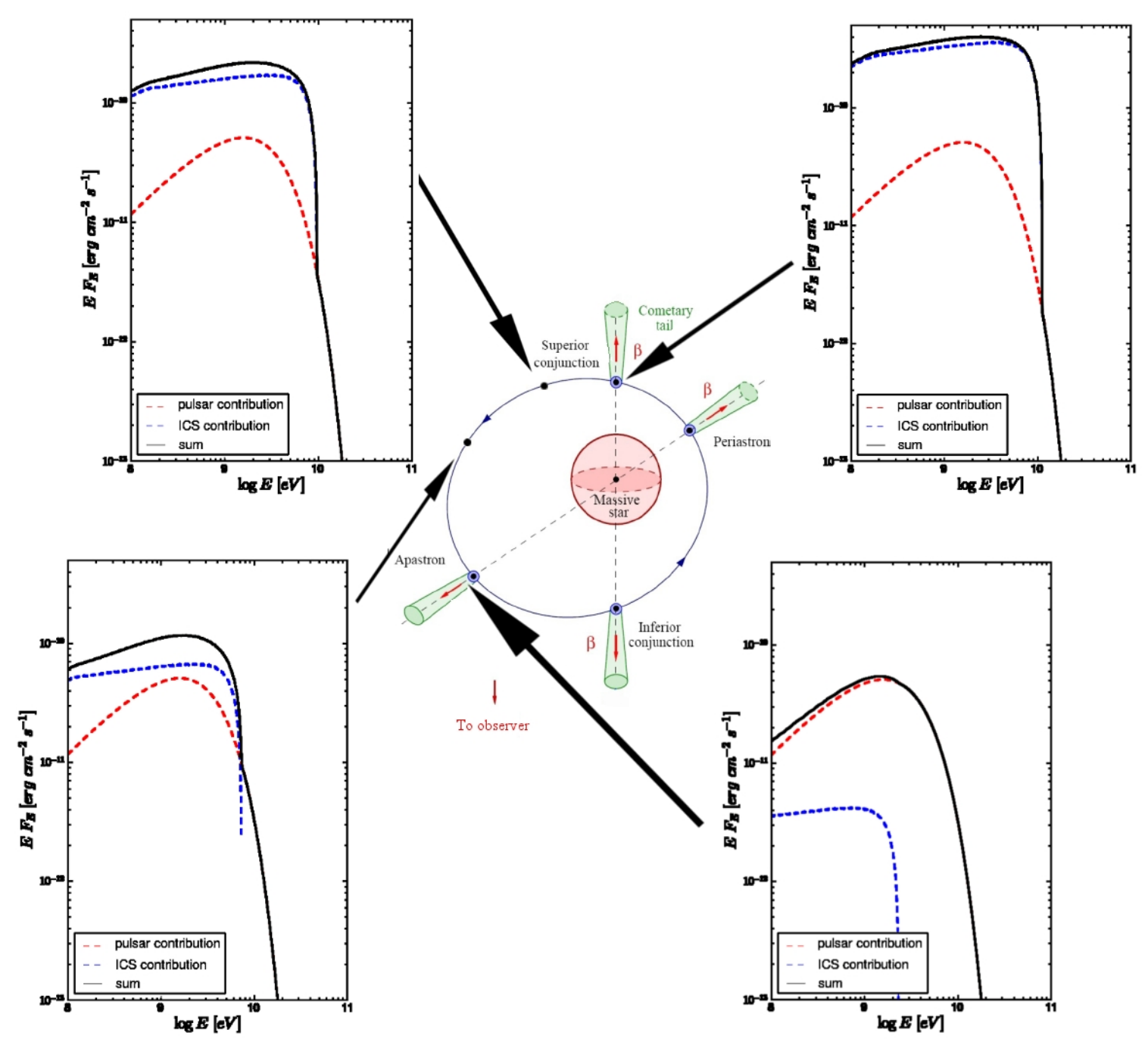

Figure 3: The orbit LS 5039 is to scale. The green cone shows the geometry of a collimated shock material (PWN; Dubus et al. 2010 [7]). Our model SED for indicated orbital phases (schematic picture of the system taken from Cerutti et al. (2010) [4].

of the O-star (Kapala et al. 2011 [8]).

The calculated orbital modulations of the total emission above $100 \mathrm{MeV}$ are shown in Fig. 5. The shape of light curve (top panel) is qualitatively well reproduced. However the amplitude of modulations is notably larger than the observed one (right panel). The hardness ratio (bottom panel) also reproduces main observed characteristics. More realistic assumptions, such as replacing the point-like source of soft photons with a star of finite size, will be presented in Kapala et al. (2011) [8]. 

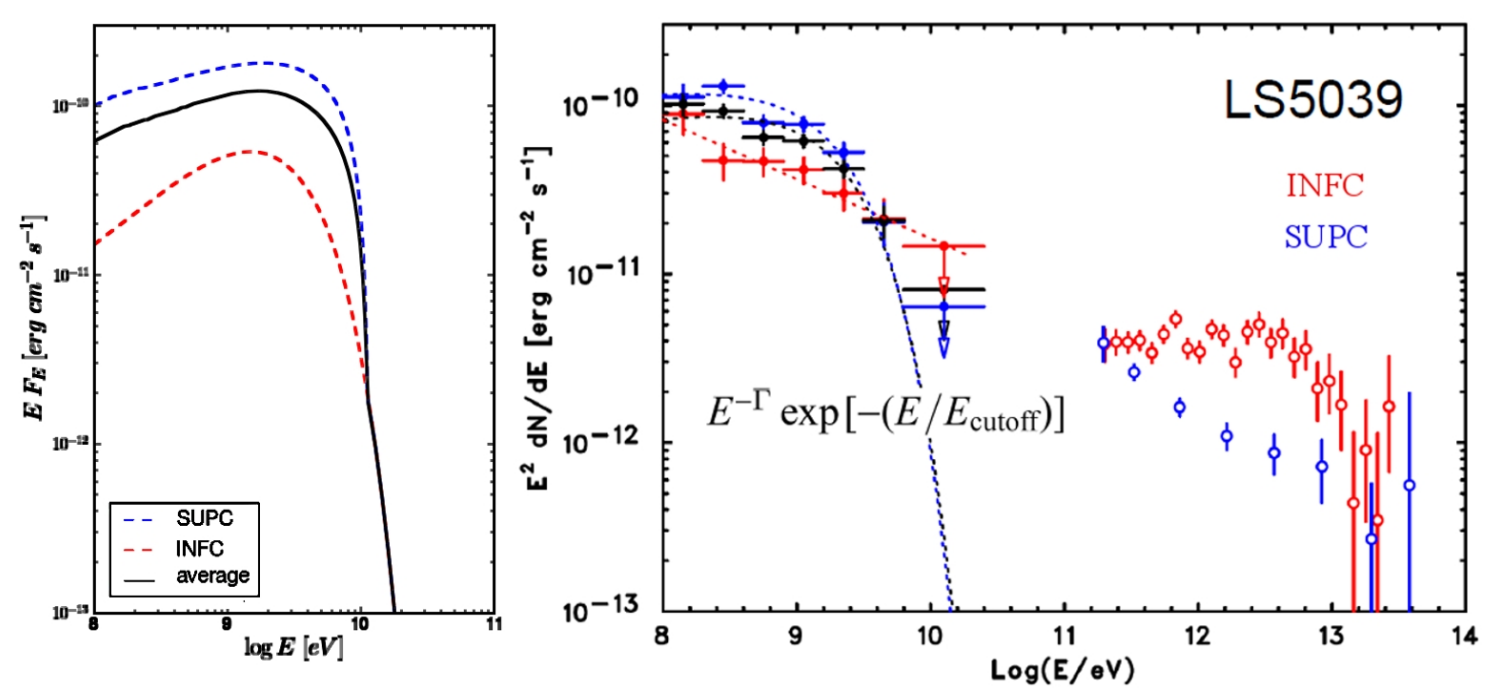

Figure 4: Left panel shows the model SED averaged over the orbital period. Right panel shows the observed SED (Fermi/LAT; Abdo et al. 2009 [1]). Black points represent phase-averaged spectrum.
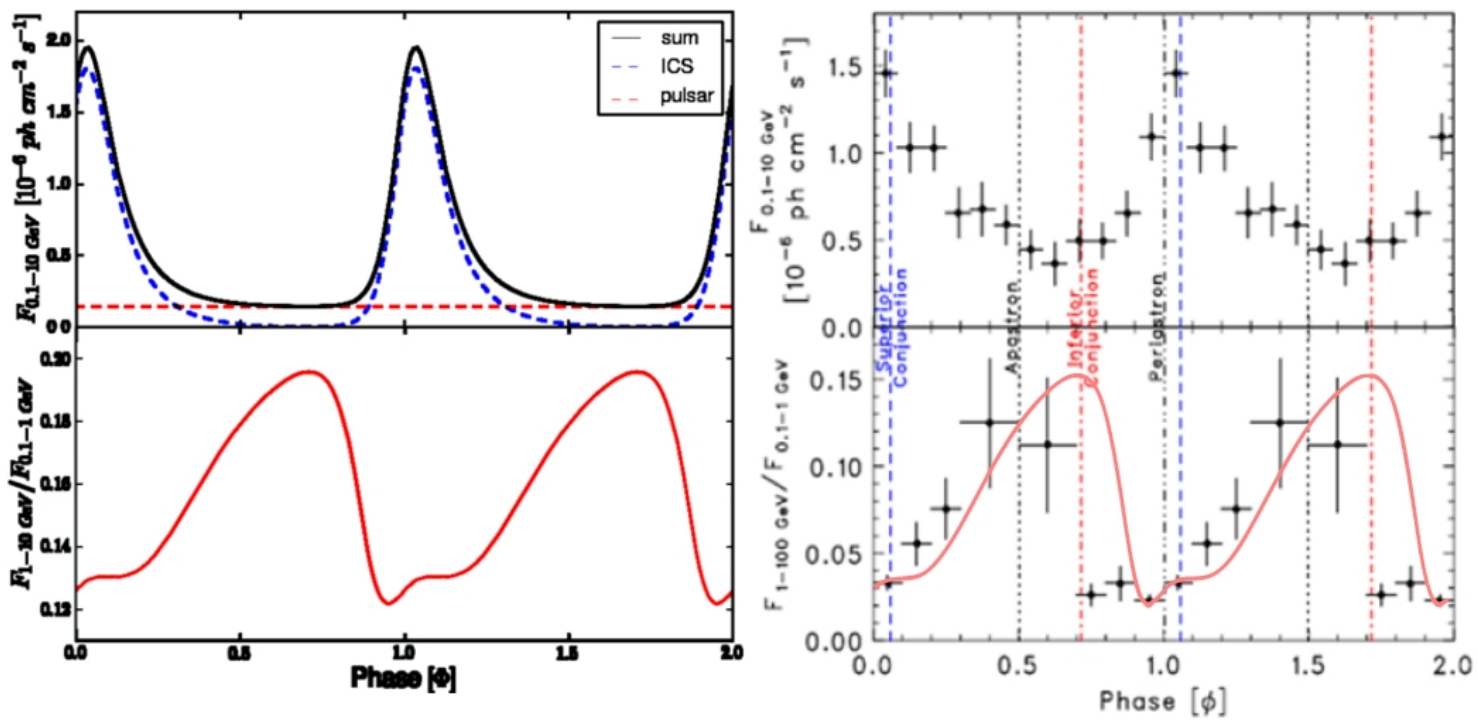

Figure 5: The comparison of the model (left) with observations by Fermi/LAT (right; Abdo et al. 2009 [1]). Top: the flux modulations vs. orbital phase for $0.1-10 \mathrm{GeV}$. Bottom: the hardness ratio modulations with orbital phase.

\section{Conclusions}

The spectral and temporal properties of the $\gamma$-ray radiation from the binary LS 5039 in the HE domain are explained by postulating a middle-aged pulsar in the system. This model of the HE radiation from LS 5039 seems to complement the recently proposed model of boosted X-ray emission from LS 5039 (Dubus et al. 2010) [7] to explain simultaneous orbital modulations of the flux in VHE (Aharonian et al. 2005) [3] and in hard X-rays (Takahashi et al. 2009) [11]. Direct 
proof of the pulsar residing in LS 5039 would come from finding pulsed emission in the Fermi LAT data. If the two-component model holds then pulsation searches in the data should be limited to the energy range around $10 \mathrm{GeV}$. The ICS photons around $100 \mathrm{MeV}$ are not expected to reveal pulsations unless the searches are limited to orbital phases roughly between the apastron passage and Inferior Conjuction.

Acknowledgements:

We acknowledge financial support by the grants N N203 387737 and N N203 302835 of the Polish Ministry of Science and Higher Education.

\section{References}

[1] A.A. Abdo et al., Fermi/LAT observations of LS 5039, ApJ, 706L, 56 [0 910 . 5520].

[2] A.A. Abdo et al., The First Fermi Large Area Telescope Catalog of Gamma-ray Pulsars, ApJS, 187, 460 [0067-0049].

[3] F. Aharonian et al., Discovery of Very High Energy Gamma Rays Associated with an X-ray Binary, Science, 309, 746 [astro-ph/ 0508298 ].

[4] B. Cerutti et al., 2010, ICREA Workshop, San Cugat.

[5] G. Dubus, Gamma-ray binaries: pulsars in disguise?, A\&A, 456, 801 [astro-ph/0605287].

[6] G. Dubus et al., The modulation of the gamma-ray emission from the binary LS 5039, A\&A,477,691 [0710.0968].

[7] G. Dubus et al., Relativistic Doppler-boosted emission in gamma-ray binaries A\&A, 516, 18 [1004.0511].

[8] M. Kapala et al., 2011 (in preparation).

[9] A. Martocchia et al., The low X-ray state of LS 5039/RX J1826.2-1450, A\&A, 430, 245 [astro-ph/0409608].

[10] B. Rudak et al., 2011 (in preparation).

[11] T. Takahashi et al., Study of the Spectral and Temporal Characteristics of X-Ray Emission of the Gamma-Ray Binary LS 5039 with Suzaku, ApJ, 697, 592 [10.1088/000 4-637X]. 